\title{
Subsidio escolar a la población vulnerable (SEP): Implementación e impacto en escuelas rurales en Chile
}

\author{
School subsidy to vulnerable populations (SEP): \\ Implementation and impact on rural schools in Chile
}

\section{Amada Opazo' \\ Claudio Frites ${ }^{2}$ \\ Leopoldo López ${ }^{3}$}

\section{Resumo}

Este trabalho corresponde a um estudo de caso que analisa a distribuição dos recursos de um dos instrumentos de correção de desigualdade, conhecida como a Lei de Subvençõ Escolar Preferencial. Para tanto, se contextualiza e descreve o funcionamento desta política pública, a partir de material administrativo e de entrevistas com diretores escolares. O trabalho conclui pontuando algumas críticas a partir de evidências empíricas que mostram que o uso desses recursos e o nível do custo/efetividade em torno de se obter bons resultados em testes de qualidade da educação chilena. Uma vez que depois de investir USD 500.000 em seis instituições de ensino rurais, logram subir marginalmente sua pontuação média em dois anos (1\%), observandose ainda algumas escolas subindo e outras baixando.

Palavras-chave: Subvenção; Equidade; Política Educacional; Política Escolar e gestão de recursos.

\section{Resumen}

Este trabajo corresponde a un estudio de caso que analiza la distribución de los recursos de uno de los instrumentos correctores de inequidad, conocido como la Ley de Subvención Escolar Preferencial. Para ello, se contextualiza y se describe la operación de esta política pública, a partir de material administrativo y de entrevistas con los directores de los establecimientos. Se concluye planteando algunas críticas a partir de la evidencia empírica insuficiente que sustenta el uso de estos recursos y nivel de costo/efectividad en torno a la obtención de buenos resultados en la prueba de calidad de la educación chilena. Puesto que después de invertir US\$ 500.000 en seis establecimientos educacionales rurales, logran subir marginalmente su puntaje promedio en dos años (1\%), con algunas escuelas que suben y otras que bajan.

Palabras claves: Subvención; equidad; política educativa; política escolar y gestión de recursos.

1 Profesora Educación Física. Instituto Investigación y Desarrollo Educacional, Universidad de Talca.

2 Sociólogo Universidad de Chile. Instituto Investigación y Desarrollo Educacional, Universidad de Talca.

3 MBA Florida International University. Instituto Investigación y Desarrollo Educacional, Universidad de Talca. 


\begin{abstract}
This work corresponds to a case study that analyzes the distribution of the resources of one of the instruments of inequity correction, known as the "Ley SEP". This analysis is done by contextualizing and describing the operation of this public policy, based on administrative material and interviews with the schools' principals. We conclude by raising some criticism about the insufficient empirical evidence to support the use of these resources and the level of cost/effectiveness in obtaining positive results in the Chilean quality test of education. After investing US\$500,000 in six schools, the test scores climbed only marginally in two years (1\%), with some schools going up and others down.
\end{abstract}

Key words: Voucher, equity, educational policy, k12 policy and resources management.

\title{
Introducción
}

En el contexto social chileno es una materia clave mejorar la calidad y equidad de la educación. En Chile, cuando ya han sido superados en grado importante los problemas de acceso y cobertura, con una educación obligatoria de 12 años y con inversión pública y privada que prácticamente se ha doblado en los últimos 20 años, la temática de la equidad es claramente, junto a la calidad la tarea del presente y del futuro.

En esta línea, se han implementado una serie de medidas insertas en diversas reformas que dan cuenta de esta preocupación, pero que no han alcanzado los resultados esperados. Dada la complejidad de los desafíos para el ámbito educativo, a saber: la ciudadanía, la equidad y la productividad. Es imperativo analizar la implementación de políticas que tienen por objetivo reducir la brecha entre los resultados y mejorar los estándares educativos.

\section{Antecedentes}

El sistema de educación en Chile experimentó profundas transformaciones desde inicios de la década de 1980, instaurándose un modelo de financiamiento, gestión y estructura, que descentraliza su administración e introdujo incentivos de mercado para estimular el aumento de escuelas privadas con financiamiento estatal (Cox, 2003). Se trató de reformas profundas de carácter estructural, para las que no hubo necesidad de consensos dada la existencia de una dictadura.

Se configuró de este modo un mercado educativo imperfecto, de gran dinámica, pero con una serie de fallas inherentes al sector en se que aplicaba, las que han sido ampliamente documentadas, ya que -entre otras razones-, las escuelas no funcionan como empresas privadas, los padres no se comportan como consumidores "racionales"; no hay información suficiente, por lo que se inhibe la competencia transparente; y por último, la educación es un bien de valor social y no solo un bien de consumo (CEPAL, 2000).

Desde entonces el financiamiento estatal es canalizado -esencialmente- a través de una subvención fija, que es administrada por los municipios cuando la provisión es pública y por sostenedores particulares cuando la provisión es privada, esquema de subvenciones que se extiende a nivel nacional y alcanza actualmente a más del 90\% de la población escolar. A lo que se agrega una tercera clase de establecimientos, los privados pagados, existentes previo a estas reformas, que no reciben financiamiento del Estado y cuya incidencia históricamente nunca ha superado el 10\% de la matrícula total (Contreras et. al, 2011).

A partir de 1990, con el advenimiento de la democracia, se diseñan e implementan una serie de políticas que se incorporan como vectores de calidad y equidad en el sistema educativo, interviniendo el sistema escolar a través de distintas reformas que implicaron: el estatuto docente, modificación de la duración de la obligatoriedad escolar, programas de intervención en salud y alimentación para estudiantes, como además en infraestructura 
y equipameinto escolar, más una reforma curricular que abarcó todo el sistema escolar. Dichas medidas significaron inversiones importantes en infraestructura, remuneraciones docentes, más el financiamiento de una serie de programas educativos (Mizala, 2009). No obstante lo anterior, las cuantiosas inversiones realizadas, que llevaron el gasto público en educación entre los años 1990 y 2008 de 2,4 a 4,2 por ciento del PIB del país (Eyzaguirre, 2010), no tuvieron el resultado esperado. De este modo, pese a los numerosos programas de apoyo y reformas impulsadas, los establecimientos educacionales han permanecido en una situación de relativo estancamiento y no han logrado repuntar en sus resultados.

En este contexto, el año 2008, se promulga la Ley de Subvención Escolar Preferencial (Ley 20.248 o Ley SEP), que entrega una subvención diferenciada -aproximadamente un 50\% adicional al subsidio basal- a los mantenedores de establecimientos escolares que adhieren a la Ley SEP. La incorporación de la escuela a esta modalidad es voluntaria, y se entrega conforme el número de estudiantes vulnerables.

La Ley SEP no establece diferencias según el tipo de dependencia (propietario o mantenedor del establecimiento sea privado o público), por su lógica puede ser vista, en primer lugar, como una corrección de la desigualdad en la asignación del "precio" de educar a estudiantes menos aventajados y, en segundo lugar, como un estímulo -un 50\% más de subvención por estudiante- que considera un aumento significativo en los resultados esperados en el SIMCE (Sistema de Información y Medición de la Calidad de la Educación) y rendición de cuentas de los establecimientos (Elacqua, 2010).

De este modo, la SEP busca mejorar el sistema educativo en dos ámbitos: desde la equidad, pues introduce elementos correctivos al prohibir prácticas de selección de ingreso de los estudiantes al establecimiento (al menos hasta el $6^{\circ}$ grado) y de expulsión; asumiendo las diferencias socioeconómicas de los estudiantes otorgando mayor financiamiento a los que definidos como prioritarios, es decir: para "quienes la situación económica dificulte sus posibilidades de enfrentar el proceso educativo" (Artículo $2^{\circ}$, Ley 20.248). En segundo lugar, desde la calidad, al implicar compromisos y consecuencias de acuerdo al logro de metas y resultados observables (Rivera, 2007).

Previo a la Ley SEP, por espacio de 28 años el financiamiento del sistema escolar utilizó como herramienta principal un subsidio plano que al tenor de todos los actores relevantes ha reforzado las desigualdades existentes (Sapelli, 2006), dado que su monto variaba solo en relación a sus costos de operación -de acuerdo a su nivel, modalidad de enseñanza y ubicación geográfica- (Mineduc, 2007) -algo así como un 7\% del valorsin considerar el nivel socioeconómico de los estudiantes (González, Mizala y Romaguera, 2002). En la medida que no se considera el mayor costo de educar a los estudiantes más vulnerables, se presenta un incentivo a la exclusión y segregación, que se materializaba a través de procesos de selección y expulsión desde el sistema privado hacia el sistema público, que está obligado, como corresponde, a aceptar a todos los estudiantes como parte del derecho a educación que confiere la sociedad por intermedio del Estado. No existiendo tampoco una posibilidad real de escoger las escuelas para los padres ("school choice"), en la medida que operaban estos procesos, reforzados por el financiamiento compartido, que es un cobro adicional que pueden realizar los establecimeintos escolares, especialmente los privados, sin que ello implique perder el subsidio por estudiante que reciben del Estado (Donoso, 2011).

De este modo, buscando corregir este problema de diseño del sistema de financiamiento instalado desde el año 1981, el año 2008 se promulga la Ley SEP (Ley 20.248), que entrega una subvención adicional -pasando de US\$ 75 a $120^{5}$ por estudiante- para quienes son identificados como prioritarios, que se definen según su vulnerabilidad socioeconómica y por su nivel de concentración al interior de los establecimientos.

4 EI SIMCE es una prueba anual y estandarizada, los peores establecimientos obtienen puntajes del orden de 210 mientras que los mejores del orden de 320 puntos.

5 En US\$ al 31de julio de 2012 donde U $\$ 1,00$ equivale a $\$ 483,00$ pesos chilenos. 
La Ley SEP se establece que un estudiante es prioritario si cumple al menos uno de los siguientes requisitos:

Pertenecer al programa Chile Solidario (política de gobierno que trabaja con la extrema pobreza).

Se encuentra en el tercio más vulnerable según la ficha de protección social, que evalúa el grado de vulnerabilidad de las familias, a través de indicadores cualitativos y cuantitativos.

Pertenecer al tramo A del Fondo Nacional de Salud (FONASA).

Si el hogar tiene bajos ingresos familiares, baja escolaridad de la madre, ruralidad o pobreza comunal.

Una exigencia de la Ley SEP se refiere a la elaboración de Planes de Mejoramiento que deben diseñar y ejecutar los establecimientos escolares beneficiarios de este subsidio, condición que además es perentoria para poder adjudicarse los fondos. Los Planes deben abordar dimensiones como gestión del currículo, liderazgo escolar, convivencia escolar y gestión de recursos 6 . Estos procesos de mejora son apoyados por organismos externos privados que trabajan con y sin fines de lucro -las denominadas Asesorías Técnica Educativa (ATE)-, que elaboran Planes de Mejoramiento educativo en las escuelas. Con ese fin, se ha creado un registro público de personas y entidades pedagógicas y técnicas de apoyo, que están habilitadas para prestar asesoría técnica a los establecimientos educacionales (Bellei: 2010). En la tabla siguiente se presentan las dimensiones susceptibles de inversión con un detalle no exhaustivo de los ítemes en los que se pueden destinar los recursos.

Tabla N 1: Categorías de Gastos de la Ley SEP

\begin{tabular}{|c|c|c|c|c|c|c|}
\hline $\begin{array}{l}1^{\circ} \text { Categoría: } \\
\text { Gastos de } \\
\text { Operación } \\
\text { de bienes y } \\
\text { servicios }\end{array}$ & $\begin{array}{c}2^{\circ} \text { Categoría: } \\
\text { Gastos en } \\
\text { Personal }\end{array}$ & $\begin{array}{c}3^{\circ} \text { Categoría: } \\
\text { Gastos en } \\
\text { asesoría técnica } \\
\text { y capacitación }\end{array}$ & $\begin{array}{l}4^{\circ} \text { Categoría: } \\
\text { Gastos en } \\
\text { Recursos de } \\
\text { Aprendizaje }\end{array}$ & $\begin{array}{l}5^{\circ} \text { Categoría: } \\
\text { Gastos en } \\
\text { equipamiento } \\
\text { de apoyo } \\
\text { pedagógico. }\end{array}$ & $\begin{array}{l}6^{\circ} \text { Categoría: } \\
\text { Gastos de } \\
\text { imprevistos }\end{array}$ & $\begin{array}{l}\text { Categoría } \\
\text { Especial: Gastos } \\
\text { de Zona de } \\
\text { Catástrofe }\end{array}$ \\
\hline $\begin{array}{l}\text { Materiales } \\
\text { escolares }\end{array}$ & $\begin{array}{l}\text { Horas docentes } \\
\text { por subsector }\end{array}$ & $\begin{array}{c}\text { Contratación de } \\
\text { una Asistencia } \\
\text { Técnica Educativa } \\
\text { ATE }\end{array}$ & $\begin{array}{c}\text { Talleres de apoyo } \\
\text { al lenguaje }\end{array}$ & Textos de estudio & $\begin{array}{l}\text { Papel de } \\
\text { impresión }\end{array}$ & Mobiliario \\
\hline $\begin{array}{c}\text { Materiales de } \\
\text { Oficina }\end{array}$ & $\begin{array}{l}\text { Horas docentes } \\
\text { para coordinación }\end{array}$ & $\begin{array}{c}\text { Cursos de } \\
\text { perfeccionamiento } \\
\text { docente }\end{array}$ & $\begin{array}{l}\text { Talleres de apoyo } \\
\text { a las matemáticas }\end{array}$ & Cuentos & Repuestos & $\begin{array}{l}\text { Reparaciones } \\
\text { Menores }\end{array}$ \\
\hline $\begin{array}{c}\text { Materiales de } \\
\text { bibliotecas }\end{array}$ & $\begin{array}{l}\text { Horas asistentes } \\
\text { de la educación }\end{array}$ & $\begin{array}{l}\text { Contratación de } \\
\text { asistencia de } \\
\text { perfeccionamiento }\end{array}$ & $\begin{array}{l}\text { Talleres de } \\
\text { convivencia }\end{array}$ & Literatura & $\begin{array}{c}\text { Cafetería / Coffe } \\
\text { Break }\end{array}$ & Material de Aseo \\
\hline $\begin{array}{c}\text { Materiales } \\
\text { tecnológicos }\end{array}$ & $\begin{array}{l}\text { Horas asistentes } \\
\text { administrativos }\end{array}$ & $\begin{array}{c}\text { Cursos de } \\
\text { capacitación }\end{array}$ & $\begin{array}{l}\text { Talleres de } \\
\text { liderazgo }\end{array}$ & Pizarras & $\begin{array}{l}\text { Archivadores- } \\
\text { carpetas }\end{array}$ & $\begin{array}{l}\text { Material de } \\
\text { Construcción }\end{array}$ \\
\hline Arriendo de salas & Horas monitores & $\begin{array}{l}\text { Asistentes de la } \\
\text { educación }\end{array}$ & $\begin{array}{l}\text { Talleres de } \\
\text { autoestima }\end{array}$ & Computadores & Convivencias & $\begin{array}{l}\text { Horas de } \\
\text { carpintería }\end{array}$ \\
\hline
\end{tabular}

Fuente: Elaboración propia

La Ley SEP se implementa en su primer año desde prekínder (PK) hasta cuarto grado básico (4), a la que se incorporan, de manera gradual y sucesiva, un curso por año, hasta completar la enseñanza secundaria (12 años en total).

En su implementación, las escuelas que adhieren a la Ley SEP son clasificadas según su rendimiento en la prueba SIMCE en "autónomas o emergentes". Las primeras son aquellas que presentan buenos resultados, mientras las segundas deben diseñar e implementar un programa de mejoramiento educativo, que les permita realizar mejoras en cuatro años. Si después de ese periodo no se obtienen los resultados deseados, esa escuela es declarada "en recuperación", y se otorga en reemplazo de la Subvención Escolar Preferencial un aporte transitorio, mientras se implementa un nuevo plan, en el que participan sostenedor, MINEDUC y una entidad

6 Dimensiones consideradas en el Marco para la Buena Dirección, que orienta desde el año 2005 el hacer de los establecimientos escolares en esta materia. 
técnica reconocida. A su vez, los estándares nacionales y criterios específicos para la calificación de resultados, son actualizados cada cinco años por el Ministerio de Educación (Artículo 10).

Dada la existencia de un sistema altamente heterogéneo, la autoridad opera bajo el supuesto que desde una perspectiva central es muy difícil saber qué hace a una escuela mejor que otra y, por tanto, sería imposible determinar pautas generales, o qué procesos de aprendizaje son los más efectivos para cada una de ellas. Respecto a este esquema, el supuesto es que el rol de la autoridad es indicar los incentivos correctos que motiven a las escuelas a adoptar planes y políticas para mejorar sus resultados pedagógicos. Esa es la lógica que subyace a la Ley SEP, dado que asume la complejidad de los procesos de aprendizaje y que algunas metodologías o programas funcionan mejor en algunos lugares que otros (dada la existencia, por ejemplo, de factores socioeconómicos o étnicos).

La investigación que se expone evaluó la implementación de esta Ley en seis establecimientos educacionales rurales de la región del Maule, en particular en qué ámbitos se han invertido los recursos SEP dispuestos. Además, se compara el uso de recursos por escuela y se contrasta con el aumento del puntaje en la prueba SIMCE para los cuartos básicos.

La importancia del estudio reside en que se observa que la planificación se ha dejado en el espacio local y sin existir evidencia concluyente o consenso respecto a cuáles son las mejores decisiones de inversión en el uso de recursos o respecto a cuál es la relación entre costo y efectividad en los resultados. Es relevante analizar en qué ámbitos los colegios han destinado sus recursos y el posible impacto implementado estas reformas.

\section{Metodología}

Se evaluaron seis establecimientos escolares con alto índice de vulnerabilidad y de ruralidad en la Región del Maule, perteneciente al territorio centro sur del país (comunas de Sagrada Familia y Curepto), que se incorporaron a la Ley de Subvención Escolar Preferencial (SEP) desde el primer año de funcionamiento el 2008.

Se obtuvo información de los registros de la Ley SEP y de entrevistas a los directores, respecto de la inversión del año 2009 y 2010, cuando se operacionaliza de manera estable la Ley en esas escuelas.

Los establecimientos educacionales se seleccionaron de manera intencionada, atendiendo a que tuvieran una alta matrícula de estudiantes vulnerables, existieran tanto escuelas emergentes como autónomas, estuvieran ubicadas en el sector rural y un alto presupuesto dedicado a Planes de Mejoramiento.

\section{Caracterización de la muestra}

Las escuelas serán identificadas mediante números, las tres primeras $-\mathrm{N}^{\circ} 1, \mathrm{~N}^{\circ} 2$ y $\mathrm{N}^{\circ}$ 3- serán las correspondientes a la Comuna de Sagrada Familia y las tres últimas $-\mathrm{N}^{\circ} 4$, № 5 y N ${ }^{\circ} 6$ - a la Comuna de Curepto.

Tabla №2: Escuelas que participan en el estudio

\begin{tabular}{|c|c|c|c|c|c|}
\hline Escuela & Clasificación & Matrícula & \% Prioritarios & SIMCE 2008 & Grupo \\
\hline 1 & Autónoma & 461 & $48 \%$ & 257 & Medio-Bajo \\
\hline 2 & Emergente & 173 & $49 \%$ & 269,5 & Medio-Bajo \\
\hline 3 & Autónoma & 340 & $64 \%$ & 292 & Medio-Bajo \\
\hline 4 & Autónoma & 536 & $30 \%$ & 248 & Medio Bajo \\
\hline 5 & Emergente & 102 & $51 \%$ & 244 & Bajo \\
\hline 6 & Emergente & 60 & $67 \%$ & 213 & Bajo \\
\hline
\end{tabular}




\section{Análisis y Resultados}

El objetivo de la investigación fue identificar la distribución de los recursos financieros de la Subvención Escolar Preferencial de seis escuelas con altos índices de vulnerabilidad pertenecientes a dos comunas rurales del territorio centro sur del país (Región del Maule ${ }^{7}$ ). Los datos dan cuenta que no hay un patrón definido y que los distintos énfasis corresponden a las categorías de: (i) inversión de materiales de insumos de bienes y servicios, así como de (ii) equipamientos de apoyo pedagógico.

\section{Gastos SEP por año}

En la tabla $N^{\circ} 3$ se muestra el resultado anual de gastos, marcándose una alta diferencia de inversión para los años en estudio. Quizás lo más relevante desde el punto de vista financiero es que las escuelas no invirtieron todo el dinero que tenían destinado, las razones pueden: cautela en la inversión, que el Ministerio de Educación envía dinero al final del año escolar, los que son entegrados a los mantenedores (o sostenedores) municipales, demorándose la transferencia a las escuelas. Cualquiera sea el caso, esto redunda en menor efectividad en el uso de los recursos.

Tabla N³: Resultados gastos SEP (US\$)

\begin{tabular}{|c|c|c|c|}
\hline Escuelas & $\mathbf{2 0 0 9}$ & $\mathbf{2 0 1 0}$ & Total \\
\hline 1 & $38.693,08$ & $124.078,66$ & 162.772 \\
\hline 2 & $17.425,77$ & $44.529,55$ & 61.955 \\
\hline 3 & $42.396,15$ & $44.445,47$ & 86.842 \\
\hline 4 & $20.465,14$ & $120.771,82$ & 141.237 \\
\hline 5 & $13.604,73$ & $18.456,30$ & 32.061 \\
\hline 6 & $6.512,76$ & $29.537,94$ & 36.051 \\
\hline
\end{tabular}

Nota: Gastos SEP por año

Las escuelas en estudio reciben ingresos diferenciados, en consideración al número de estudiantes prioritarios y su nivel de concentración. Las escuelas $\mathrm{N}^{\circ} 1$ y $\mathrm{N}^{\circ} 4$ tienen los más altos gastos, mientras las $\mathrm{N}^{\circ} 2$, $N^{\circ} 5$ y $N^{\circ} 6$ tienen los menores. La escuela $N^{\circ} 3$, es la que presenta un gasto con menos diferencias entre el año 2009 y 2010.

Cuatro de las seis escuelas (menos la $N^{\circ} 3$ y $N^{\circ} 5$ ) tienen altas diferencias de inversión SEP de un año a otro, esto puede ser por el excedente que dejaron y se invierte el segundo año, o bien porque su propuesta de inversión siguió ese patrón. La cantidad de dinero que se invierte en el año 2009, es mucho menor que la del año 2010, sin que la matrícula de estudiantes prioritarios sea muy distinta de un año a otro, cuestión que es llamativa y que no se sabe a qué razón responde.

En el siguiente cuadro se muestra los recursos que reciben por año las escuelas del estudio. Este dato tiene que ver con el Ingreso de recursos SEP por año. 
Tabla N4: Ingresos SEP por Escuela (US\$)

\begin{tabular}{|c|c|c|c|}
\hline Escuelas & $\mathbf{2 0 0 9}$ & $\mathbf{2 0 1 0}$ & TOTAL \\
\hline $\mathbf{1}$ & 111.600 & 123.476 & 235.075 \\
\hline $\mathbf{2}$ & 42.125 & 44.812 & 86.938 \\
\hline $\mathbf{3}$ & 81.359 & 99.322 & 180.681 \\
\hline $\mathbf{4}$ & 117.289 & 119.421 & 236.710 \\
\hline $\mathbf{5}$ & 22.414 & 16.012 & 38.425 \\
\hline $\mathbf{6}$ & 16.958 & 20.781 & 37.739 \\
\hline
\end{tabular}

Nota: Ingresos de las escuelas por concepto SEP. Son los registros que informan los sostenedores

Los ingresos de las escuelas por concepto de la Ley SEP son similares de un año a otro y levemente mayores en el segundo año. Esto quiere decir que las matrículas por estudiantes vulnerables se mantienen del primer y segundo año o se incrementan levemente. De este modo, se puede proyectar la inversión SEP por los cuatro años que será implementado el Plan de Mejoramiento de cada escuela, con una mayor certidumbre respecto a la estabilidad de los ingresos en el tiempo.

Cada escuela recibe un monto anual por los estudiantes prioritarios que asisten a la escuela y sobre ese monto deben trabajar para invertir en su Plan de Mejoramiento. A continuación se presenta el nivel de ejecución presupuestaria, a considerar en los años 2009 y 2010.

Tabla N5: Ingresos egresos SEP año 2009-2010 US\$

\begin{tabular}{|c|c|c|c|c|}
\hline Escuela & Total de Ingresos & \% de Ingresos & Total de Egresos & $\%$ de Egreso \\
\hline $\mathbf{1}$ & 235.075 & $100 \%$ & 162.772 & $69,24 \%$ \\
\hline $\mathbf{2}$ & 86.938 & $100 \%$ & 61.955 & $71,26 \%$ \\
\hline $\mathbf{3}$ & 180.681 & $100 \%$ & 86.842 & $48,06 \%$ \\
\hline $\mathbf{4}$ & 236.710 & $100 \%$ & 141.237 & $59,67 \%$ \\
\hline $\mathbf{5}$ & 38.425 & $100 \%$ & 32.061 & $83,44 \%$ \\
\hline $\mathbf{6}$ & 37.739 & $100 \%$ & 36.051 & $95,53 \%$ \\
\hline
\end{tabular}

Nota: Ingresos v/s Egresos

Las escuelas acogidas a la Ley SEP presentan una gran variabilidad en los niveles de ejecución presupuestaria -entre un $48 \%$ y un $95.5 \%$ - dando cuenta de una ineficiencia en el uso de recursos, pues registran un saldo que se acumula para al año siguiente. Las escuelas gastan menos de lo que reciben, lo que da cuenta de una gestión que debe mejorar, lo que puede deberse a inversiones subestimadas y/o problemas de coordinación entre los municipios y el MINEDUC, ya que los recursos llegan muy tarde y habría en ocasiones una gestión deficiente. Este último punto se está mejorando -parcialmente- dada la obligación de que los municipios tengan una cuenta separada para los dineros SEP.

\section{Gastos SEP por Categorías}

Se presentan las categorías en las que se invierte de acuerdo al Plan de Mejoramiento. En la siguiente tabla se resumen de gastos por categoría: 
Tabla N6: Resumen de Gastos por Categorías años 2009-2010 (US\$)

\begin{tabular}{|c|c|c|c|c|c|c|}
\hline Categorías & Escuela 1 & Escuela 2 & Escuela 3 & Escuela 4 & Escuela 5 & Escuela 6 \\
\hline Gastos de operación de bienes y servicios & $45,04 \%$ & $42,20 \%$ & $73,55 \%$ & $23,44 \%$ & $53,39 \%$ & $62,28 \%$ \\
\hline Gastos en personal & $17,94 \%$ & $25,76 \%$ & $8,11 \%$ & $0,00 \%$ & $0,00 \%$ & $0,00 \%$ \\
\hline Gastos en asesoría técnica y capacitación & $8,50 \%$ & $15,73 \%$ & $12,10 \%$ & $0,00 \%$ & $22,89 \%$ & $11,89 \%$ \\
\hline Gastos en Recursos de Aprendizaje & $0,00 \%$ & $0,00 \%$ & $0,00 \%$ & $0,00 \%$ & $1,59 \%$ & $2,52 \%$ \\
\hline Gastos en equipamiento de apoyo pedagógico & $27,58 \%$ & $15,59 \%$ & $6,19 \%$ & $68,33 \%$ & $14,20 \%$ & $5,60 \%$ \\
\hline Gastos de imprevistos & $0,82 \%$ & $0,46 \%$ & $0,04 \%$ & $0,00 \%$ & $0,00 \%$ & $17,72 \%$ \\
\hline Gastos de zona de catástrofe (categoría especial) & $0,12 \%$ & $0,25 \%$ & $0,00 \%$ & $8,23 \%$ & $7,92 \%$ & $0,00 \%$ \\
\hline Total & $100,00 \%$ & $100,00 \%$ & $100,00 \%$ & $100,00 \%$ & $100,00 \%$ & $100,00 \%$ \\
\hline
\end{tabular}

Fuente: Elaboración propia

Nota: Gastos por categorías agrupados en el año 2009 y 2010 según registros de las escuelas y /o sostenedor

La tabla $N^{\circ} 6$ expone el total de recursos invertidos en cada categoría para implementar los Planes de Mejoramiento Educativo. Una primera observación se refiere a la priorización en bienes-servicios, asesoría técnicacapacitación, y equipamiento de apoyo pedagógico. En la categoría de bienes y servicios mientras la escuela $\mathrm{N}^{\circ}$ 4 invierte un $23,4 \%$ de sus recursos, la escuela $N^{\circ} 3$ destina un $73,5 \%$ de sus dineros SEP. Asimismo, vemos este amplio rango en las inversiones en equipamiento de apoyo pedagógico, donde la escuela que menos invierte es la $N^{\circ} 6$ con un 5,6\% mientras la que más lo hace es la escuela $N^{\circ} 4$ con un 68,3\%. La heterogeneidad en el uso de recursos da cuenta de la libertad en la elaboración de los Planes de Mejoramiento, las distintas realidades que presentan los establecimientos y las percepciones que los directores tienen sobre sus realidades.

\section{Entrevistas Directivos}

Se realizaron entrevistas para evaluar la percepción de los directivos respecto a la inversión de los recursos de la SEP, en cuanto a: el Plan de Mejoramiento, sus acciones y los objetivos que se persiguen con los recursos. Los directores llevan registro de la administración de los recursos SEP, de manera de permitir el monitoreo de las acciones de su Plan de Mejoramiento. Esto se materializa a través de registros impresos a los que se tuvo acceso, cumpliendo de este modo con la exigencia de transparencia en la administración de los recursos, existiendo respaldo de todos los ingresos y egresos de la inversión de recursos en su escuela, que permite el monitoreo constante y accountability de los gastos en resguardo de los recursos de los más pobres.

Superado lo administrativo-contable, se evalúan las acciones de Plan de Mejoramiento y la percepción respecto a la lógica que subyace a la toma de decisiones, atendiendo a la equidad para la calidad educativa.

En términos generales, se alude a compras de materiales tecnológicos, para mejora del lenguaje, matemáticas y ciencias. Aumento de horas docentes, textos, incorporación de otros profesionales de apoyo - psicólogos, fonoaudiólogos, psicopedagogos, etc.-, giras de estudios, insumos en papel y tinta.

De este modo, la mayoría de los establecimientos trabaja tomando los recursos y atendiendo sus necesidades inmediatas de materiales básicos para su funcionamiento. Una excepción corresponde a la escuela №3, cuyo director expresa que sus compras son parte de un proyecto estratégico, que enfatiza la atención a los estudiantes prioritarios, que asistan a la escuela sin tener ningún gasto y cubrir todas las necesidades educativas, que incluye vestuario, útiles escolares y transporte. 
En lo referente a la relación entre estas decisiones de inversión SEP y las mejoras en los resultados, se señala que hay evaluaciones mayoritariamente externas que velan por eso, ATE, SIMCE y monitoreo del MINEDUC (Ministerio de Educación). Externalizando de este modo la evaluación sobre la pertinencia de las inversiones.

Al consultar sobre la percepción de la calidad de los aprendizajes con las medidas adoptadas por el programa de Subvención Preferencial. Se señala preferentemente las áreas del currículo, como el mejoramiento en los subsectores de matemáticas y lenguaje, y la posibilidad de involucrar a los apoderados y mejorar la convivencia. El cambio se valora de manera favorable, en la medida que se puede implementar adecuadamente en cada escuela, pero plantean reparos sobre la burocracia para la adquisición de los materiales. No obstante, en general los directores están conformes con las inversiones, y los resultados de mejora los establecen con el SIMCE, evaluaciones con ATE y monitoreos periódicos.

En cuanto a la participación y sugerencias, se señala la necesidad de mejorar el apoyo del MINEDUC y la excesiva burocracia del sistema de implementación de las acciones, marcada, en alguna medida, por la inexperiencia al gestionar recursos directamente.

Al momento de establecer sugerencias a otros directivos, se plantea que es necesario focalizar la inversión, especialmente en equipos multidisciplinarios, y capacitaciones. Asimismo, se busca que la definición de los destinos de los recursos sea más autónoma o al menos compartida y no solo quede supeditada a lo que plantee el DAEM (Dirección de Administración Educativa Municipal).

Respecto a la implementación de la Ley SEP en sus establecimientos, las reacciones son variadas: en algunas escuelas los docentes lo vieron como una oportunidad, para otros fue una complicación debido a las nuevas tareas que imponía, un tercer grupo la rechazó inicialmente y luego fue mejorando su nivel de compromiso.

Las escuelas implementan sus Planes de manera muy mediática, con poca participación y como ellos mismos manifiestan contra el tiempo y sin espacios de reflexión, además con muy poca capacitación para la gestión y administración de recursos en un contexto deprimido y con solo la "esperanza" de movilidad educativa en razón a la mejora.

Respecto a la relación entre la inversión declarada en los Planes y las expectativas de calidad que sustenta esta subvención en las metas establecidas, se señala que existe esta relación, pero no es tan claro cuál es en definitiva la correspondencia de causalidad, por la dispersión del uso de los recursos y la novedad de la autogestión, y las exigencias de objetivos de aprendizajes dados por el Ministerio de Educación.

Las inversiones realizadas dejan ver las debilidades de la burocracia que comparten los sostenedores con el MINEDUC. Esto se refiere concretamente, a que las acciones y adquisiciones se demoran y atrasan la implementación del Plan de Mejoramiento, sobre todo los compromisos de horas con distintos profesionales. En este sentido, la gran incertidumbre debilita la autonomía que se propone como uno de sus principios. A esto se agrega, en ocasiones, la falta de compromiso de los docentes para apoyar con confianza este nuevo proyecto de escuela, en consideración a experiencias anteriores y por miedo a las consecuencias asociadas a los resultados esperados de parte de las autoridades, que son vistas como una amenaza.

Respecto a la focalización en los estudiantes prioritarios, en algunos casos las inversiones han sido directamente en su beneficio, aunque no siempre se reducen a ellos, por ende impactan a toda la escuela, y en algunos casos a toda la familia de los estudiantes, puesto que la implementación del vestuario, de útiles y de transporte antes era toda responsabilidad de la familia, aunque no pudiera cumplir sus obligaciones por sus bajos ingresos. Sin embargo, también hay casos en que los recursos adquiridos no estaban en uso -embalados o en bodegas- para evitar su deterioro y los estudiantes no tenían acceso. Debido a que no existía una persona encargada de administrar o procurar por su buen uso, lo que pone el acento en que junto a la inversión, se debe considerar la instalación de nuevas capacidades, junto a la mantención y sostenibilidad de la intervención.

Por ello es relevante contemplar dentro del plan la forma en que se usarán los recursos, y administrarán los recursos teniendo claros los protocolos de uso y de ejecución de todas las acciones. 


\section{Registro de la percepción de motivación, expectativa y/o acogida}

Los directores tienen una percepción de exceso de responsabilidad ante las demandas de la Ley SEP. En el caso de la escuela $N^{0} 1$, el director manifiesta buena acogida en general de la puesta en marcha de esta nueva iniciativa, son más recursos, pero sin mayor énfasis en el aporte específico que debe significar a la igualdad y equidad para los estudiantes prioritarios. No domina número de matrícula ni las especificaciones de los ingresos por ese concepto.

El director de la escuela $N^{\circ} 2$ mantiene la información y organiza las acciones ordenadamente, pero ve con algo de temor la responsabilidad de manejar los recursos contra resultados. El director de la escuela № 3 está muy informado de la propuesta SEP y manifiesta un gran entusiasmo en esta nueva posibilidad de mejora para su escuela y especialmente hacia los estudiantes prioritarios, se preocupa de que todos tengan sus materiales escolares y uniformes, entre otras implementaciones.

El director de la escuela $N^{\circ} 4$ es neutro en su opinión y tiene poco orden en cuanto a sus recursos SEP, pero tiene altos ingreso por este concepto pues es una escuela grande. El de la escuela $N^{\circ} 5$ manifiesta desmotivación y bajas expectativas de la implementación de la Ley. Dice que es rechazada por los profesores. Finalmente.el director de la escuela $N^{0} 6$ muestra mucho entusiasmo por los nuevos recursos para los estudiantes. Mantiene alta motivación aunque sostiene críticas por la poca fluidez del trabajo en conjunto con el sostenedor y el Ministerio de Educación.

Por lo tanto, los directores de las escuelas manifiestan distintas percepciones en relación con la Ley SEP, unos muy motivados, otros muy descontentos y otros con pocos anhelos, pero funcionando.

\section{Los Resultados SIMCE}

Los resultados de Calidad de los Aprendizajes son controlados a través del SIMCE instrumento de evaluación de la calidad educativa en Chile, y que permite evaluar cuánto mejoran en los aprendizajes las escuelas en estudio. En la actualidad el SIMCE se da en $4^{\circ}$ y $8^{\circ}$ básico. Se considera solo los resultados del nivel de $4^{\circ}$ básico, pues la Ley en el periodo analizado solo impacta a estos estudiantes.

\section{Gráfico N 1: Variación Puntaje SIMCE 2008 - 2010}

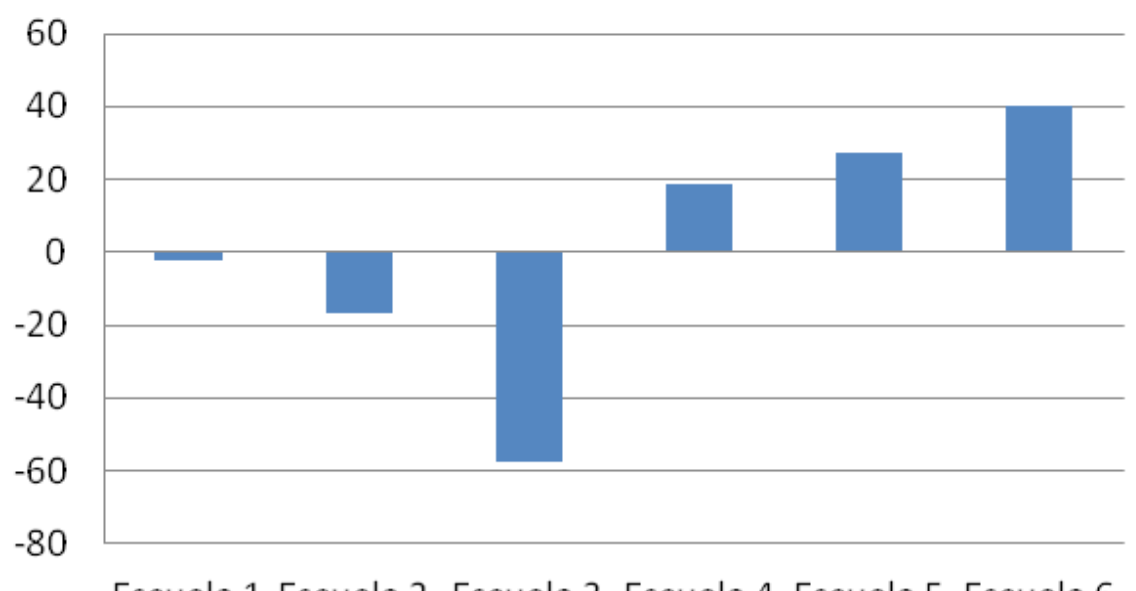

Escuela 1 Escuela 2 Escuela 3 Escuela 4 Escuela 5 Escuela 6

Fuente: www.simce.cl

Como se desprende del gráfico, tres escuelas bajan sus puntajes y otras logran subir, en promedio los seis establecimientos subieron 1,66 puntos con una inversión total de US\$ 520.917. 


\section{Gráfico N2: Variación puntaje SIMCE versus monto total invertido US\$}

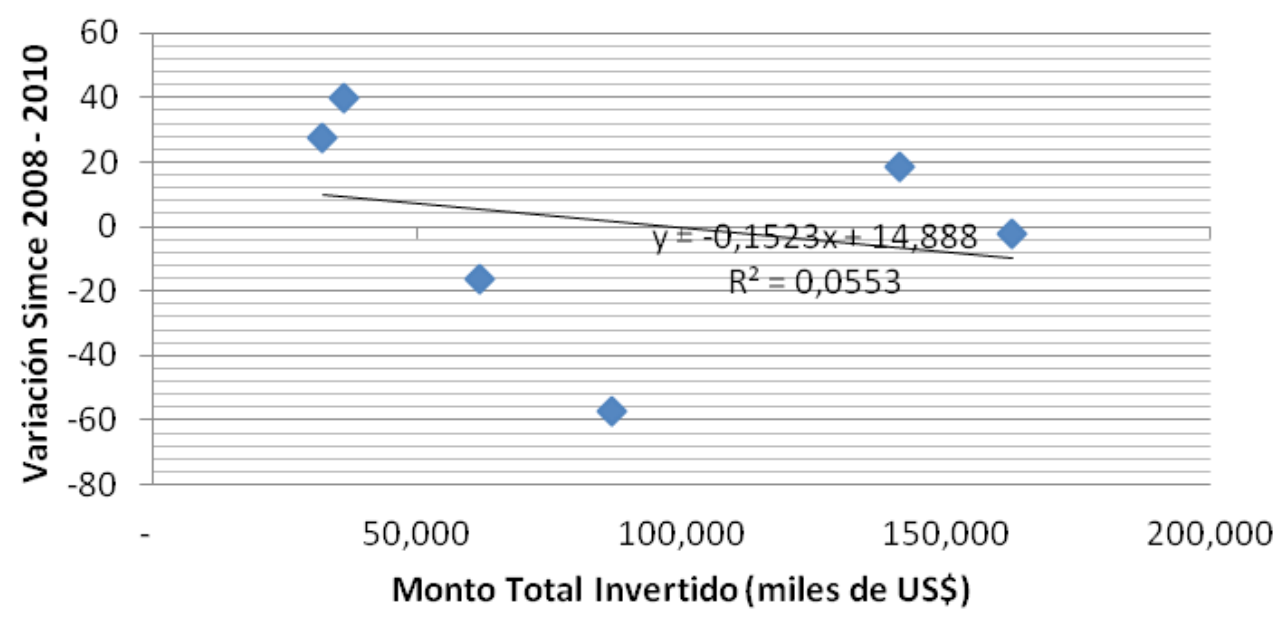

Fuente: Elaboración propia

Al graficarse la variación del puntaje SIMCE en relación al monto total invertido, se obtiene un coeficiente de correlación de -0,24, lo que implica que aunque no hay una fuerte la relación, a mayor inversión menor son los resultados. Sin embargo el $\mathrm{R}^{2}$ indicaría que la relación entre monto invertido y aumento de puntaje marginal.

\section{Gráfico N 3: Variación puntaje SIMCE 2008 - 2010 versus Inversión por estudiante prioritario}

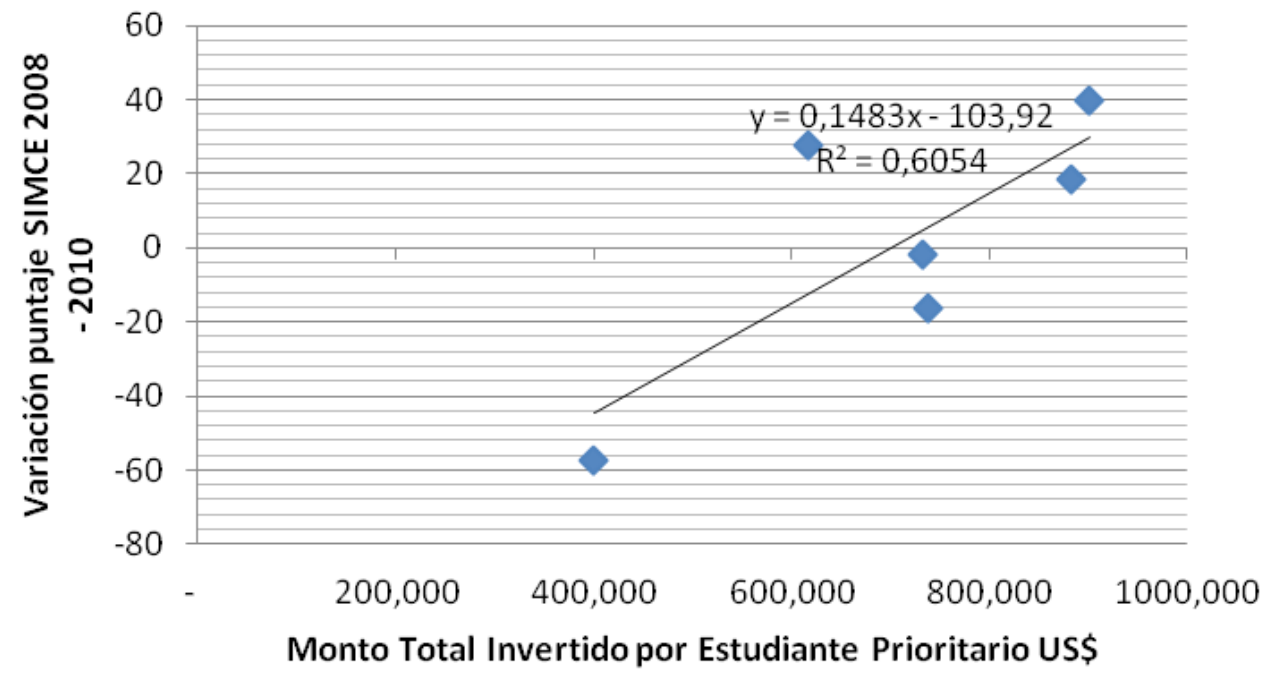

Fuente: Elaboración propia

El gráfico muestra la relación positiva entre dinero invertido por estudiante prioritario y la variación del puntaje SIMCE. Aquí se puede apreciar una correlación de 0,78. Sin embargo se está suponiendo que todo el dinero va destinado a estudiantes prioritarios lo que no siempre es válido.

\section{Conclusiones}

En primer lugar la Ley SEP se inserta como un vector de calidad y equidad en el sistema, dado que reconoce por primera vez que es más costoso educar a los estudiantes más vulnerables. Sin embargo el impacto de esta 
Ley es difícil de medir ya que el destino de los nuevos recursos no permite observar una focalización en los estuantes prioritarios, las escuelas han invertido en gastos generales como el mejoramiento de la infraestructura que favorece a toda la comunidad educativa. Por los resultados obtenidos podemos afirmar que las inversiones no han tenido efecto en lo pedagógico. Por otro lado es difícil medir el impacto de la Ley SEP en los estudiantes vulnerables ya que no se entregan los resultados SIMCE pormenorizados.

El diseño de la Ley SEP plantea una evaluación de la política del gobierno cada cuatro años, no obstante el análisis de la implementación en las seis escuelas estudiadas, dan cuenta de la necesidad de un seguimiento y monitoreo permanente por parte de la autoridad para incorporar correcciones oportunas respecto a la efectividad de los gastos realizados y la esperanza de mejoras en lo pedagógico a partir de esta inversión.

Con respecto a la implementación de la Ley, se constata un bajo nivel de ejecución del presupuesto, esta situación se puede deber a dificultades de diversa índole: problemas administrativos, temor a la fiscalización o poca claridad en torno a las exigencias en la rendición de cuentas, pueden haber llevado a una excesiva prudencia en el uso de los recursos. Cualquiera sea el caso, esto revela que a la hora de la implementación no habían equipos capacitados adecuadamente para estos fines y asumir los desafíos de estas nuevas reformas.

A partir de esta situación, surge la interrogante sobre la focalización y efectividad de los recursos invertidos. Focalización, pensando en la mejora del rendimiento de aquellos estudiantes que son definidos como prioritarios y efectividad, cuando se observan los resultados obtenidos y se comparan con los esperados.

En general los establecimientos no son capaces de diagnosticar sus problemas pedagógicos y no tienen una retroalimentación cotidiana de sus procesos de aprendizajes.

De este modo, los criterios para utilizar los recursos se presentan de manera poco clara, aparentemente sin un proceso de discusión o una base empírica. Esto es muy complejo, dado que el eje de esta política debiera ser exclusivamente lo pedagógico y estos recursos presentan una serie de usos alternativos. Sin haber un debate o una discusión relevante sobre estos procesos, es válida la sospecha sobre hasta qué punto se puede seguir aumentando los recursos, sin que esto tenga su correlato en los resultados académicos. Si bien este estudio aborda solo 6 establecimientos, es importante ampliar la investigación.

Por último se observa que los directores tienen la percepción que están utilizando de buena forma los recursos SEP, sin que halla existido por parte de las autoridades ni de las ATEs una retroalimentación que los haga salir de ese estado de autocomplacencia.

\section{Bibliografía}

Bellei, Cristián; González, Clemencia (2010). "La asistencia técnica educativa en Chile y sus factores de calidad. Una revisión de la literatura". Asistencia técnica educativa: de la intuición a la evidencia. Fondef.

CEPAL (2000). "Equidad, desarrollo y ciudadanía”. CEPAL- Naciones Unidas.

Contreras, Dante; Hojman, Daniel; Huneeus, Federico, Landerretche, Óscar (2011). "El lucro en la educación escolar. Evidencia y Desafíos Regulatorios." Trabajos de Investigación en Políticas Públicas. Departamento de Economía Universidad de Chile.

Cox, Cristián (2003) "Las políticas educacionales de Chile en las últimas dos décadas del siglo XX". En Políticas educacionales en el cambio de siglo. La reforma del sistema escolar de Chile. Cristián Cox et al. Editorial Universitaria.

Donoso, Sebastián (2011); "El Financiamiento de la Educación Pública Chilena vía Subsidio: Consecuencias críticas sobre el Rol del Estado en Educación”. FINEDUCA. Revista de Financiamiento da Educaçao, Vol. 1. Revista electrónica, disponible en http://seer.ufrgs.br/fineduca

Elacqua, Gregory; Mosqueira, Úrsula; Santos, Humberto (2010) "La toma de decisiones de un sostenedor; 
Análisis a partir de la ley SEP”. Expansiva UDP, Instituto de Políticas Públicas.

Eyzaguirre, Sylvia (2010). "Hacia reformas de segunda generación en educación”. Puntos de Referencia N³27. Centro de Estudios Públicos.

González, Pablo; Mizala, Alejandra; Romaguera, Pilar (2002). "Recursos diferenciados a la educación subvencionada en Chile”. Documentos de Trabajo Serie Economía. Nro. 150.

Mizala, Alejandra (2007). "La economía política de la reforma educacional en Chile”. Serie Estudios Socio / Económicos $N^{\circ}$ 36. Cieplan.

Mizala, Alejandra (2009). "Vouchers educativos en Chile, la experiencia de un cuarto de siglo". En J. Diaz Malledo (ed) Financiación de la Enseñanza Obligatoria: los Bonos Escolares en la Teoría y en la Práctica". Fundación Funcas, Madrid. Colección Estudios de la Fundación.

Rivera, Felipe (2007). "Proyecto de Ley sobre Subvención Escolar Preferencial para niños y Niñas socioEconómicamente Vulnerables". Biblioteca del Congreso Nacional de Chile.

MINEDUC (2005). “Marco para la buena dirección”. División de Educación General. Ministerio de Educación.

MINEDUC (2007). "Guía de Subvenciones Educacionales". Departamento de Estudios y Desarrollo. División de Planificación y Presupuesto. Ministerio de Educación.

Ley No 20.248 (2008). "Ley de Subvención Escolar Preferencial”. Biblioteca del Congreso Nacional de Chile.

Sapelli, Cristián (2006) "Desafíos del sistema educativo: La subvención preferencial” en Temas de Agenda Pública (Santiago de Chile: Pontificia Universidad Católica de Chile, Vicerrectoría de Comunicaciones y Asuntos Públicos) Año 1, № 1. 